\title{
Combination of Nanomaterials and Organic Semiconductors for Electronic and Optoelectronic Device Fabrication
}

\author{
Ricardo Izquierdo \\ Department of Electrical Engineering, Ecole de Technologie Superieure \\ Canada
}

The use of a combination of nanomaterials and organic semiconductors offers a great flexibility for the fabrication of various electronic and optoelectronic devices. In this talk, we will present various types of device, which were obtained by using such a combination. First device is an organic memory consists of two aluminium electrodes separated by a layer from an organic semiconductor that contains gold nanoparticles supported by parylene pillars. A second example is the use of carbon nanotubes or graphene for semi-transparent conducting electrodes for electroluminescent organic diodes (OLEDs). We will also discuss about the efficiency enhancement of a standard bulk heterojunction (BHJ) solar cell by the incorporation of various nanomaterials such as a nanohybrid composite material made of lead sulfide ( $\mathrm{PbS}$ ) colloidal quantum dots (QDs) and multiwall carbon nanotubes (MWCNTs) or by the incorporation of gold nanorods. Finally, the combination of a semitransparent electrochemical sensor, made from silver nanowires, and a fluorescence sensor, made from organic optoelectronic components, in order to build a sensing platform for water toxicity evaluation, will be presented. 